\title{
DIRECT DECOMPOSITION OF TENSOR PRODUCTS INTO SUBTENSOR PRODUCTS
}

\author{
I. Y. CHUNG
}

\begin{abstract}
A subtensor product of a family of modules is defined by using a subdirect product of the family of modules considered as sets. A tensor product of modules can be decomposed into a direct sum of subtensor products of the modules. Subtensor products of graded modules and graded algebras are also studied. As an application of these, a certain subtensor product of a family (not necessarily finite) of anticommutative algebras is shown to be a coproduct of this family in the category of unitary anticommutative algebras, and it can be imbedded as a direct summand into a tensor product of the family as modules.
\end{abstract}

1. Subtensor product of modules. Let $\left(M_{\alpha}\right)_{\alpha \in I}$ be a family of modules over a commutative ring $R$ with unit, and $U$ a subset of the cartesian product $\prod_{\alpha \in I} M_{\alpha}$ as sets. For an $R$-module $N$, a mapping $\varphi: U \rightarrow N$ will be called a multilinear mapping of $U$ into $N$ if for any $\left(x_{\alpha}\right)_{\alpha \in I},\left(y_{\alpha}\right)_{\alpha \in I}$ and $\left(z_{\alpha}\right)_{\alpha \in I}$ in $U$ such that $x_{\beta}=\lambda y_{\beta}+\mu z_{\beta}$, where $\lambda$ and $\mu$ are in $R$, for one $\beta \in I$, and $x_{\alpha}=y_{\alpha}=z_{\alpha}$ for all $\alpha \in I$ with $\alpha \neq \beta$,

$$
\varphi\left(\left(x_{\alpha}\right)_{\alpha \in I}\right)=\lambda \varphi\left(\left(y_{\alpha}\right)_{\alpha \in I}\right)+\mu \varphi\left(\left(z_{\alpha}\right)_{\alpha \in I}\right)
$$

holds. A multilinear mapping of $\prod_{\alpha \in I} M_{\alpha}$ into $N$ is a usual multilinear mapping; and a restriction mapping of this to $U$ is an example of a multilinear mapping in our sense. By a similar construction to that of a tensor product of modules [1, Theorem 37, p. 87], the following theorem can be proved.

THEOREM 1. For any $U \subseteq \prod_{\alpha \in I} M_{\alpha}$, there exist an $R$-module $A$ and $a$ multilinear mapping $\sigma$ of $U$ into $A$ such that for any $R$-module $N$ and for any multilinear mapping $\varphi$ of $U$ into $N$ there exists a unique linear mapping $f$ of $A$ into $N$ such that $f \circ \sigma=\varphi$.

Proof. Let $F$ be a free $R$-module with $U$ as its basis; and $J$ be the submodule of $F$ generated by the elements $\left(x_{\alpha}\right)_{\alpha \in I}-\lambda\left(y_{\alpha}\right)_{\alpha \in I}-\mu\left(z_{\alpha}\right)_{\alpha \in I}$, where

Presented to the Society, January 21, 1972; received by the editors September 28, 1971 and, in revised form, February 28, 1972.

AMS (MOS) subject classifications (1969). Primary 1580; Secondary 1610, 1590.

Key words and phrases. Tensor product, subtensor product, graded module, graded algebra, anticommutative algebra, coproduct, category.

(c) American Mathematical Society 1973 
$\left(x_{\alpha}\right)_{\alpha \in I},\left(y_{\alpha}\right)_{\alpha \in I}$ and $\left(z_{\alpha}\right)_{\alpha \in I}$ are in $U, \lambda, \mu \in R$, such that $x_{\beta}=\lambda y_{\beta}+\mu z_{\beta}$ for one $\beta \in I$, and $x_{\alpha}=y_{\alpha}=z_{\alpha}$ for all $\alpha \in I$ with $\alpha \neq \beta$. Put $A=F / J$, and let $\sigma$ be the restriction mapping of the natural homomorphism of $F$ onto $A$ to $U$. Q.E.D.

Definitions. Let $U \subseteq \prod_{\alpha \in I} M_{\alpha}$ be a subdirect product, i.e., for any $\beta \in I$, the mapping $U \rightarrow M_{\beta},\left(x_{\alpha}\right)_{\alpha \in I} \mapsto x_{\beta}$ is onto. A pair $(A, \sigma)$, where $A$ and $\sigma$ are as in Theorem 1 , will be called a subtensor product of $\left(M_{\alpha}\right)_{\alpha \in I}$ with respect to a subdirect product of $\left(M_{\alpha}\right)_{\alpha \in I}$. A will be denoted by $\otimes_{\alpha \in I} M_{\alpha}$; and $\sigma\left(\left(x_{\alpha}\right)_{\alpha \in I}\right)$ by ${ }_{U} \otimes x_{\alpha}$, or ${ }_{U} \bigotimes_{\alpha \in I} x_{\alpha} . f$ is called the linearization of $\varphi$.

REMARK 1. Let $\left(N_{\alpha}\right)_{\alpha \in I}$ be a family of $R$-modules such that $M_{\alpha}$ is a submodule of $N_{\alpha}$ for each $\alpha \in I$. If a subtensor product were defined for an arbitrary subset $U$ of $\prod_{\alpha \in I} M_{\alpha}$, i.e., $U$ is not necessarily a subdirect product, then ${ }_{U} \otimes_{\alpha \in I} M_{\alpha} \cong U \bigotimes_{\alpha \in I} N_{\alpha}$. This we do not want.

As usual, a subtensor product of $\left(M_{\alpha}\right)_{\alpha \in I}$ with respect to $U$ is uniquely determined up to unique isomorphisms, and it is generated by the set of all elements $U \otimes x_{\alpha}$ such that $\left(x_{\alpha}\right)_{\alpha \in I} \in U$.

2. Direct decompositions into subtensor product. Let $U$ be a subset of $\prod_{\alpha \in I} M_{\alpha}$ satisfying the following condition (2.1).

(2.1) For any $\left(x_{\alpha}\right)_{\alpha \in I},\left(y_{\alpha}\right)_{\alpha \in I}$, and $\left(z_{\alpha}\right)_{\alpha \in I}$ in $\prod_{\alpha \in I} M_{\alpha}$ such that $x_{\beta}=$ $\lambda y_{\beta}+\mu z_{\beta}, \lambda, \mu \in R$, for one $\beta \in I$, and $x_{\alpha}=y_{\alpha}=z_{\alpha}$ for all $\alpha \in I$ with $\alpha \neq \beta$, if $\left(x_{\alpha}\right)_{\alpha \in I}$ is in $U$, both $\left(y_{\alpha}\right)_{\alpha \in I}$ and $\left(z_{\alpha}\right)_{\alpha \in I}$ are in $U$.

Then $U$ is a subdirect product of $\left(M_{\alpha}\right)_{\alpha \in I}$. In fact, let $\left(x_{\alpha}\right)_{\alpha \in I} \in U$. For any $y \in M_{\beta}$, let $\left(y_{\alpha}\right)_{\alpha \in I}$ be an element of $\prod_{\alpha \in I} M_{\alpha}$ such that $y_{\beta}=y$ and $y_{\alpha}=x_{\alpha}$ for all $\alpha \neq \beta ;\left(z_{\alpha}\right)_{\alpha \in I}$ such that $z_{\beta}=x_{\beta}-y$ and $z_{\alpha}=x_{\alpha}$ for all $\alpha \neq \beta$. Then $\left(y_{\alpha}\right)_{\alpha \in I} \in U$ by (2.1). It follows that the mapping $U \rightarrow M_{\beta},\left(x_{\alpha}\right)_{\alpha \in I} \mapsto x_{\beta}$ is onto. Hence $U$ is a subdirect product.

TheOREM 2. Suppose that $\prod_{\alpha \in I} M_{\alpha}=\bigcup_{j \in J} U_{j}$ be a partition of $\prod_{\alpha \in I} M_{\alpha}$ into mutually disjoint subsets $U_{j}$ satisfying (2.1) for each $j \in J$. Let $S_{j}$ be the submodule of $T=\otimes_{\alpha \in I} M_{\alpha}$ generated by the elements $\otimes x_{\alpha}$ such that $\left(x_{\alpha}\right)_{\alpha \in I} \in U_{j}$. Then

(1) $T=\sum_{j \in J} S_{j}$ (direct),

(2) $S_{j} \cong U_{j} \otimes_{\alpha \in I} M_{\alpha}$, for each $j \in J$.

More precisely, the linear mapping $q_{j}:_{U_{j}} \otimes_{\alpha \in I} M_{\alpha} \rightarrow T, U, \otimes x_{\alpha} \mapsto \otimes x_{\alpha}$, is one-to-one and onto $S_{j}$.

Proof. Let $q: \oplus_{j \in J}\left(U_{j} \otimes_{\alpha \in I} M_{\alpha}\right) \rightarrow T$ be the linear mapping defined by $q\left(\left(y_{j}\right)_{j \in J}\right)=\sum_{j \in J} q_{j}\left(y_{j}\right)$, where $y_{j} \in_{U_{j}} \otimes_{\alpha \in I} M_{\alpha}$. Also, for each $i \in J$, let $p_{i}: U_{i} \rightarrow \oplus_{j \in J}\left(U_{j} \otimes_{\alpha \in I} M_{\alpha}\right)$ be the multilinear mapping defined by $p_{i}\left(\left(x_{\alpha}\right)_{\alpha \in I}\right)=\left(y_{j}\right)_{j \in J}$ such that $y_{i}={U_{i}}_{i} \otimes x_{\alpha}$ and $y_{j}=0$ for all $j \neq i$. Let $p^{\prime}: \prod_{\alpha \in I} M_{\alpha} \rightarrow \bigoplus_{j \in J}\left(U_{j} \otimes_{\alpha \in I} M_{\alpha}\right)$ be the mapping extending each $p_{i}$, $i \in J . p^{\prime}$ is a multilinear mapping, since every $U_{j}$ satisfies (2.1). If $p$ is the 
linearization of $p^{\prime}$, then $q \circ p$ and $p \circ q$ are identity mappings. Hence $q$ is an isomorphism. Also, it is clear that $q_{j}\left(U_{j} \otimes_{\alpha \in I} M_{\alpha}\right)=S_{j}$. (1) and (2) are immediate consequences of these. Q.E.D.

EXAMPLE 1. Define an equivalent relation on $\prod_{\alpha \in I} M_{\alpha}$ by $\left(w_{\alpha}\right)_{\alpha \in I} \sim$ $\left(w_{\alpha}^{\prime}\right)_{\alpha \in I}$ iff $w_{\alpha}=w_{\alpha}^{\prime}$ for almost all $\alpha \in I$. Let $\Phi=\prod_{\alpha \in I} M_{\alpha} / \sim$, the quotient set. Then every $W \in \Phi$ satisfies (2.1). Hence, if we denote by $S_{W}$ the submodule of $T$ generated by the elements $\otimes x_{\alpha}$ such that $\left(x_{\alpha}\right)_{\alpha \in I} \in W$, for each $W \in \Phi$, it follows from Theorem 2 that

$$
\begin{aligned}
T & =\sum_{W \in \Phi} S_{W} \quad(\text { direct }) ; \\
S_{W} & \cong W \bigotimes_{\alpha \in I} M_{\alpha}, \quad \text { for each } W \in \Phi .
\end{aligned}
$$

LEMMA. Let $U$ be a subset of $\prod_{\alpha \in I} M_{\alpha}$; and let $\Phi^{\prime}=\{W \in \Phi: W \cap U \neq \varnothing\}$, where $\Phi$ is as in Example 1. The following are equivalent.

(1) $U$ satisfies (2.1).

(2) $U=\bigcup_{W \in \Phi}, W$.

(3) $U^{\prime}=\prod_{\alpha \in I} M_{\alpha}-U$, the complementary set of $U$ in $\prod_{\alpha \in I} M_{\alpha}$, satisfies (2.1).

Proof. Let $\left(w_{\alpha}\right)_{\alpha \in I} \in W \cap U$. Then for any $\left(x_{\alpha}\right)_{\alpha \in I} \in W, x_{\alpha}=w_{\alpha}$ for almost all $\alpha \in I$; say, $x_{\alpha_{i}} \neq w_{\alpha_{i}}$ for $i=1,2, \cdots, n$, and $x_{\alpha}=w_{\alpha}$ for all $\alpha \in I$ with $\alpha \neq \alpha_{i}$ for $i=1,2, \cdots, n$. Let $\left(y_{\alpha}\right)_{\alpha \in I} \in \prod_{\alpha \in I} M_{\alpha}$ such that $y_{\alpha_{1}}=x_{\alpha_{1}}, y_{\alpha}=w_{\alpha}$ for all $\alpha \in I$ with $\alpha \neq \alpha_{1} ;\left(z_{\alpha}\right)_{\alpha \in I}$ such that $z_{\alpha_{1}}=w_{\alpha_{1}}-x_{\alpha_{1}}$, $z_{\alpha}=w_{\alpha}$ for all $\alpha \in I$ with $\alpha \neq \alpha_{1}$. Then $w_{\alpha}=y_{\alpha}+z_{\alpha}$ for one $\alpha=\alpha_{1}$, and $w_{\alpha}=y_{\alpha}=z_{\alpha}$ for all $\alpha \neq \alpha_{1}$. If $U$ satisfies (2.1), $\left(y_{\alpha}\right)_{\alpha \in I} \in U$. Continue this process for $\alpha_{2}, \cdots, \alpha_{n}$. It follows from this that $\left(x_{\alpha}\right)_{\alpha \in I} \in U$. This implies that $W \subseteq U$ for all $W \in \Phi^{\prime}$. Hence (1) implies (2). It is obvious that (2) implies (1). Similarly, we can prove that (2) if and only if (3).

THEOREM 3. Let $I=\bigcup_{k \in K} J_{k}$ be a partition of $I$ into mutually disjoint sets $J_{k} ; \pi_{k}: \prod_{\alpha \in I} M_{\alpha} \rightarrow \prod_{\alpha \in J_{k}} M_{\alpha}$ a mapping defined by $\pi_{k}\left(\left(x_{\alpha}\right)_{\alpha \in I}\right)=$ $\left(x_{\alpha}\right)_{\alpha \in J_{k}} ;$ and $\tau_{k}: \prod_{\alpha \in J_{k}} M_{\alpha} \rightarrow \bigotimes_{\alpha \in J_{k}} M_{\alpha}$ the universal multilinear mapping. Let $U$ be a subset of $\prod_{\alpha \in I} M_{\alpha}$ satisfying $(2.1) ; U_{k}=\pi_{k}(U) ; G_{k}=\tau_{k}\left(U_{k}\right)$; and $V=\left\{\left(y_{k}\right)_{k \in K} \in \prod_{k \in K^{K}}\left({ }_{C_{k}} \otimes_{\alpha \in J_{k}} M_{\alpha}\right): y_{k} \in G_{k}\right.$ for almost all $\left.k \in K\right\}$. Then the $R$-homomorphism

(2.2) $\theta:{ }_{U} \otimes_{\alpha \in I} M_{\alpha} \rightarrow_{V} \otimes_{k \in K}\left(U_{k} \otimes_{\alpha \in J_{k}} M_{\alpha}\right)$, such that

$$
{ }_{U} \otimes_{\alpha \in I} x_{\alpha} \mapsto{ }_{V} \otimes_{k \in K}\left(U_{k} \otimes_{\alpha \in J_{k}} x_{\alpha}\right)
$$

is an isomorphism.

Proof. Let $F_{k}$ be the free $R$-module with $U_{k}$ as basis, for which $U_{k} \bigotimes_{\alpha \in J_{k}} M_{\alpha}$ is a quotient in its construction (cf. Theorem 1), $v_{k}: F_{k} \rightarrow$ $U_{k} \bigotimes_{\alpha \in J_{k}} M_{\alpha}$ the natural homomorphism, and $S_{k}$ the kernel of $v_{k}$. We first 
consider the case where $U \in \Phi$ ( $\Phi$ as in Example 1). Let

and let

$$
\bar{U}=\left\{\left(z_{k}\right)_{k \in K} \in \prod_{k \in K} F_{k}: z_{k} \in U_{k} \text { for almost all } k \in K\right\}
$$

$$
f: \tau \bigotimes_{k \in K} F_{k} \rightarrow{ }_{V} \otimes_{k \in K}\left(U_{k} \otimes_{\alpha \in J_{k}} M_{\alpha}\right)
$$

be the linearization of $\bar{U} \rightarrow_{V} \otimes_{k \in K}\left(U_{k} \otimes_{\alpha \in J_{k}} M_{\alpha}\right),\left(z_{k}\right)_{k \in K} \mapsto V \otimes_{k \in K} v_{k}\left(z_{k}\right)$. Let $S$ be the submodule of $\tau_{\ell^{*}} \otimes_{k \in K^{-}} F_{k}$ generated by the elements $\otimes_{k \in K} z_{k}$, where, for at least one $k \in K, z_{k} \in S_{k}$. Then $S \subseteq \operatorname{Ker} f$ clearly. We will show that $S=\operatorname{Ker} f$. Let $\bar{U} \otimes z_{k}, \bar{v} \otimes z_{k}^{\prime} \in \bar{U}_{\bar{U}} \otimes_{k \in K} F_{k}$ such that $z_{k}-z_{k}^{\prime} \in S_{k}$ for all $k \in K$. Since $U \in \Phi$ and $z_{k}, z_{k}^{\prime} \in U_{k}$ for almost all $k \in K, z_{k}=z_{k}^{\prime}$ for almost all $k \in K$. Let $z_{k_{i}} \neq z_{k_{i}}^{\prime}$ for $i=1, \cdots, n$. Then ${ }_{0} \otimes z_{k}-\sigma_{0} \otimes z_{k}^{\prime}=\sum_{i=1}^{n} w_{i}$, where $w_{i}={ }_{i} \otimes_{k \in K} v_{k} ; v_{k}=z_{k}=z_{k}^{\prime}$ for $k \neq k_{1}, \cdots, k_{n} ; v_{k_{j}}=z_{k_{j}}$ if $j<i, v_{k_{i}}=z_{k_{i}}-$ $z_{k_{i}}^{\prime}, v_{k_{j}}=z_{k_{j}}^{\prime}$ if $j>i$. Since each $w_{i} \in S,{ }_{\tilde{U}} \otimes z_{k}-\tilde{v} \otimes z_{k}^{\prime} \in S$. It follows from this that $V \rightarrow{ }_{0} \otimes_{k \in K} F_{k} / S,\left(y_{k}\right)_{k \in K^{\prime}} \mapsto \bar{V}^{-} \otimes z_{k}+S$, where $z_{k} \in v_{k}^{-1}\left(y_{k}\right)$ for all $k \in K$, is a well-defined multilinear mapping. Let

$$
g: \nu \bigotimes_{k \in K}\left(U_{k} \otimes_{\alpha \in J k} M_{\alpha}\right) \rightarrow \sigma \bigotimes_{k \in K} F_{k} / S
$$

be the linearization of this, then $g \circ f=v$, where $v$ is the natural homomorphism of $\tilde{U} \otimes_{k \in K} F_{k}$ onto $\tilde{U} \otimes_{k \in K} F_{k} / S$. Hence $\operatorname{Ker} f=S$. Any element $z_{k} \in F_{k}$ can be uniquely expressed as $\sum_{i_{k}} r_{i_{k}}\left(x_{i_{k}, \alpha}\right)_{\alpha \in J_{k}}$, where $r_{i_{k}} \in R$ and $\left(x_{i_{k}, \alpha}\right)_{\alpha \in J_{k}} \in \prod_{\alpha \in J_{k}} M_{\alpha}$. Consider

$$
\bar{U} \rightarrow{ }_{U} \otimes_{\alpha \in I} M_{\alpha}, \quad\left(z_{k}\right)_{k \in K^{-}} \mapsto \sum\left(\prod_{k} r_{i_{k}}\right)_{0} \otimes_{\alpha \in I} x_{i k, \alpha},
$$

where the sum is over all $\left(i_{k}\right)_{k \in K}$. Then this mapping is properly defined, since $z_{k} \in U_{k}$ for almost all $k \in K$. Let $h$ be the linearization of this. If $z_{k} \in S_{k}$,

$$
z_{k}=\sum \pm\left(\left(x_{\alpha}\right)_{\alpha \in J_{k}}-\left(y_{\alpha}\right)_{\alpha \in J_{k}}-\left(z_{\alpha}\right)_{\alpha \in J_{k}}\right),
$$

where $x_{\beta}=y_{\beta}+z_{\beta}$ for one $\beta \in J_{k}$ and $x_{\alpha}=y_{\alpha}=z_{\alpha}$ for all $\alpha \in J_{k}, \alpha \neq \beta$. Hence if $\left(z_{k}\right)_{k \in K} \in S, h\left(\left(z_{k}\right)_{k \in K}\right)=0$. It follows that $h(S)=0$. Thus, there exists an $R$-homomorphism $\eta ; V \otimes_{k \in K}\left(U_{k} \otimes_{\alpha \in J_{k}} M_{\alpha}\right) \rightarrow \bigotimes_{\alpha \in I} M_{\alpha}$ such that $\theta \circ \eta$ and $\eta \circ \theta$ are identity mappings. We now consider the case where $U$ is a subset of $\prod_{\alpha \in I} M_{\alpha}$ satisfying (2.1). Let $U=\bigcup_{W \in \Phi^{\prime}} W\left(\Phi^{\prime}\right.$ as in the Lemma). Then by Theorem 2, the Lemma, and the previous case,

$$
U \otimes_{\alpha \in I} M_{\alpha} \cong \bigoplus_{W \in \Phi^{\prime}}\left(W^{\prime} \otimes_{\alpha \in I} M_{\alpha}\right) \cong \bigoplus_{W \in \Phi^{\prime}}\left(V_{W} \otimes_{k \in K}\left(W_{k} \otimes_{\alpha \in J_{k}} M_{\alpha}\right)\right),
$$

where $W, W_{k}$ and $V_{W}$ correspond to $U, U_{k}$ and $V$ in the previous case of $U \in \Phi$ respectively; and isomorphisms are canonical. $w_{k} \otimes_{\alpha \in J_{k}} M_{\alpha} \subseteq$ $v_{k} \otimes_{\alpha \in J_{k}} M_{\alpha}$ and $V_{W} \otimes_{k \in K}\left(w_{k} \otimes_{\alpha \in J_{k}} M_{\alpha}\right) \subseteq_{V} \otimes_{k \in K}\left(U_{k} \otimes_{\alpha \in J_{k}} M_{\alpha}\right)$ canonically, by Theorem 2, and the Lemma. $V_{V}$ 's are mutually disjoint, and 
hence

$$
\bigoplus_{W \in \Phi^{\prime}}\left(V_{W} \otimes_{k \in K}\left(w_{k} \otimes_{\alpha \in J_{k}} M_{\alpha}\right)\right) \subseteq \bigotimes_{V} \otimes_{k \in K}\left(U_{k} \otimes_{\alpha \in J_{k}} M_{\alpha}\right)
$$

canonically. Hence ${ }_{U} \otimes_{\alpha \in I} M_{\alpha}$ can be canonically imbedded into ${ }_{V} \otimes_{k \in K}\left(U_{k} \otimes_{\alpha \in J_{k}} M_{\alpha}\right)$. This canonical imbedding can be easily seen to be $\theta$, hence $\theta$ is one-to-one. $\theta$ is clearly onto. Q.E.D.

EXAMPLE 2. Let $I=\bigcup_{k \in K} J_{k}$ be a partition of $I$ into mutually disjoint sets $J_{k}$. If $K$ is a finite set, it is well known [1, Theorem 39, p. 92] that

$$
\bigotimes_{\alpha \in I} M_{\alpha} \cong \underset{k \in K}{\otimes}\left(\underset{\alpha \in J k}{\otimes} M_{\alpha}\right)
$$

by the canonical isomorphism. However, if $K$ is infinite, the situation is slightly different. As a special case of Theorem 3, if we put $U=\prod_{\alpha \in I} M_{\alpha}$, it is easy to see that $\otimes_{\alpha \in I} M_{\alpha}$ is isomorphic to a direct summand of $\otimes_{k \in K}\left(\otimes_{\alpha \in J_{k}} M_{\alpha}\right)$.

Let $I=\bigcup_{k \in K}^{k} J_{k}=\bigcup_{k^{\prime} \in K^{\prime}} J_{k^{\prime}}^{\prime}$ be two partitions of $I$ into mutually disjoint sets $J_{k}$ and $J_{k^{\prime}}^{\prime}$ respectively. If $K$ and $K^{\prime}$ are finite sets, it is clear from the isomorphism (2.3) that

$$
\underset{k \in K}{\otimes}\left(\underset{\alpha \in J_{k}}{\otimes} M_{\alpha}\right) \cong \underset{k^{\prime} \in K^{\prime}}{\otimes}\left(\underset{\alpha \in J^{\prime} k^{\prime}}{\otimes} M_{\alpha}\right)
$$

canonically. However, if at least one of $K$ and $K^{\prime}$ are infinite, (2.4) is no longer true. Let $U$ be a subset of $\prod_{\alpha \in I} M_{\alpha}$ satisfying (2.1). Let $V$ and $U_{k}$ be as in Theorem 3, $V^{\prime}$ and $U_{k^{\prime}}^{\prime}$ be defined for the partition $\bigcup_{k^{\prime} \in K^{\prime}} J_{k^{\prime}}^{\prime}$ in the same way as $V_{k}$ and $U_{k}$ respectively.

Corollary 1. Similar to (2.4),

$$
V \bigotimes_{k \in K}\left(U_{k} \otimes_{\alpha \in J_{k}} M_{\alpha}\right) \cong V^{\prime} \otimes_{k^{\prime} \in K^{\prime}}\left({ }_{U^{\prime} k^{\prime}} \otimes_{\alpha \in J^{\prime} k^{\prime}} M_{\alpha}\right)
$$

canonically.

3. Subtensor product of graded modules. In this section, we assume that, for each $\alpha \in I, M_{\alpha}=\sum_{\gamma \in \Gamma_{\alpha}} M_{\alpha, \gamma}$ (direct) is a graded $R$-module with $\Gamma_{\alpha}$ as its set of degrees; $M_{\alpha, \gamma}$ being the submodule of homogeneous elements of degree $\gamma$. For any $f \in \prod_{\alpha \in I} \Gamma_{\alpha}=P$, let $T_{f}$ be the submodule of $T=\otimes_{\alpha \in I} M_{\alpha}$ generated by the elements $\otimes x_{\alpha}$ such that $x_{\alpha} \in M_{\alpha, f(\alpha)}$ for all $\alpha \in I$. If $I$ is finite, it is well known [1, Theorem 40, p. 94] that

and that

$$
T=\sum_{f \in P^{P}} T_{f} \quad(\text { direct })
$$

$$
T_{f}=\bigotimes_{\alpha \in I} M_{\alpha . f(\alpha)}, \quad \text { for each } f \in P
$$


Hence $T$ is a graded module with $P$ as a set of degrees. However, if $I$ is infinite, (3.1) is not necessarily true.

Let $T_{H}$ be the submodule of $T$ generated by the elements $\otimes x_{\alpha} \in T$ such that $x_{\alpha}$ is homogeneous for all $\alpha \in I$ (notice that $T_{H}$ is also generated by the elements $\otimes x_{\alpha} \in T$ such that $x_{\alpha}$ is homogeneous for almost all $\alpha \in I$ ); $T_{H^{\prime}}$ generated by the elements $\otimes x_{\alpha} \in T$ such that $x_{\alpha}$ is not homogeneous for infinitely many $\alpha \in I$. Then by Theorem 2 ,

$$
\begin{gathered}
T=T_{H}+T_{H^{\prime}} \text { (direct); } \\
T_{H} \cong{ }_{H} \otimes_{\alpha \in I} M_{\alpha} \text { and } T_{H^{\prime}} \cong H^{\prime} \otimes_{\alpha \in I} M_{\alpha},
\end{gathered}
$$

where $H=\left\{\left(x_{\alpha}\right)_{\alpha \in I} \in \prod_{\alpha \in I} M_{\alpha}: x_{\alpha}\right.$ is homogeneous for almost all $\left.\alpha \in I\right\}$, and $H^{\prime}=\left\{\left(x_{\alpha}\right)_{\alpha \in I} \in \prod_{\alpha \in I} M_{\alpha}: x_{\alpha}\right.$ is not homogeneous for infinitely many $\left.\alpha \in I\right\}$.

TheOREM 4. $T_{H}=\sum_{f \in P} T_{f}$ (direct), and $T_{f} \cong \bigotimes_{\alpha \in I} M_{\alpha, f(\alpha)}$, for each $f \in P$.

Proof. Let $p_{\alpha, f(\alpha)}: M_{\alpha} \rightarrow M_{\alpha, f(\alpha)}$ be the linear mapping such that the restriction of $p_{\alpha, f(\alpha)}$ to $M_{\alpha, \gamma}, \gamma \in \Gamma_{\alpha}$, is the identity mapping on $M_{\alpha, \gamma}$ if $\gamma=f(\alpha)$, zero for all $\gamma \neq f(\alpha)$. Suppose that $p_{f}$ is the restriction mapping of $\bigotimes_{\alpha \in I} p_{\alpha, f(\alpha)}$ to $T_{H}$, and define $p: T_{H} \rightarrow \bigoplus_{f \in P}\left(\otimes_{\alpha \in I} M_{\alpha, f(\alpha)}\right)$ by $p(x)=$ $\left(p_{f}(x)\right)_{f \in P}$ for $x \in T_{H} \cdot p_{f}(x)=0$ for almost all $f \in P$, and hence $p$ is properly defined. On the other hand, let $i_{f}: \otimes_{\alpha \in I} M_{\alpha, f(\alpha)} \rightarrow T_{H}$ be the linearization of the multilinear mapping $\prod_{\alpha \in I} M_{\alpha, f(\alpha)} \rightarrow T_{H},\left(x_{\alpha}\right)_{\alpha \in I^{\prime}} \mapsto \otimes x_{\alpha}$. Let

$$
i: \bigoplus_{f \in P}\left(\bigotimes_{\alpha \in I} M_{\alpha, f(\alpha)}\right) \rightarrow T_{I I}
$$

be a linear mapping defined by

$$
i\left(\left(y_{f}\right)_{f \in P}\right)=\sum_{f \in P} i_{f}\left(y_{f}\right), \quad y_{f} \in \underset{\alpha \in I}{\bigotimes} M_{\alpha, f(\alpha)} .
$$

$p \circ i$ and $i \circ p$ are identity mappings. Hence $p$ is an isomorphism. (1) and (2) follow from this immediately. Q.E.D.

THEOREM 5. Let $U$ be a subset of $H$ satisfying (2.1), where $H$ is as in Theorem 4. Then

$$
U \otimes_{\alpha \in I} M_{\alpha} \cong \bigoplus_{f \in P}\left(U_{f} \otimes_{\alpha \in I} M_{\alpha, f(\alpha)}\right),
$$

where $U_{f}=U \cap \prod_{\alpha \in I} M_{\alpha, f(\alpha)}$. (This is a slightly generalized form of Theorem 4.)

Proof. Essentially the same as the proof of Theorem 4.

4. Generalized definition. We would like to have a generalized definition of subtensor product so that $U_{k} \otimes_{\alpha \in J_{k}} M_{\alpha}$ (in (2.2)), ${ }_{V} \otimes_{k \in K}\left({ }_{U_{k}} \bigotimes_{\alpha \in J_{k}} M_{\alpha}\right)$ 
(in (2.2)) and $U_{f} \otimes_{\alpha \in I} M_{\alpha, f(x)}$ (in (3.3)) can be expressed as ${ }_{U} \otimes_{\alpha \in J_{k}} M_{\alpha}$, ${ }_{U} \otimes_{k \in K}\left(U \otimes_{\alpha \in J_{k}} M_{\alpha}\right)$ and ${ }_{U} \otimes_{\alpha \in I} M_{\alpha, f(\alpha)}$ respectively. If we have one such, those properties which are true for a tensor product of a finite family also are true for a subtensor product of an infinite family. Namely, (2.2), (2.5) and (3.3) are

$$
\begin{gathered}
U \bigotimes_{\alpha \in I} M_{\alpha} \cong U \bigotimes_{k \in K}\left(U \bigotimes_{\alpha \in J_{k}} M_{\alpha}\right), \\
U \bigotimes_{k \in K}\left(U \bigotimes_{\alpha \in J_{k}} M_{\alpha}\right) \cong{ }_{U} \bigotimes_{k^{\prime} \in K^{\prime}}\left(U \bigotimes_{\alpha \in J^{\prime} k^{\prime}} M_{\alpha}\right), \quad \text { and } \\
U \bigotimes_{\alpha \in I} M_{\alpha} \cong \bigoplus_{f \in \mathcal{P}^{\prime}}\left(U \bigotimes_{\alpha \in I} M_{\alpha, f(\alpha)}\right),
\end{gathered}
$$

respectively. The following definition serves the purpose.

Definition. Let $\left(M_{\alpha}\right)_{\alpha \in I}$ be a family of $R$-modules, $J \subseteq I$ a subset, $J=\bigcup_{k \in K} J_{k}$ a partition of $J$ into mutually disjoint subsets, $N_{k}$ a submodule of $\otimes_{\alpha \in J_{k}} M_{\alpha}$ for each $k \in K ; \pi_{k}: \prod_{\alpha \in I} M_{\alpha} \rightarrow \prod_{\alpha \in J_{k}} M_{k}$ a mapping defined by $\pi_{k}\left(\left(x_{\alpha}\right)_{\alpha \in I}\right)=\left(x_{\alpha}\right)_{\alpha \in J_{k}}$ for each $k \in K, \tau_{k}: \prod_{\alpha \in J_{k}} M_{\alpha} \rightarrow \bigotimes_{\alpha \in J_{k}} M_{\alpha}$ the universal multilinear mapping for each $k \in K$. Let $U$ be a subset of $\prod_{\alpha \in I} M_{\alpha}, \quad U_{k}=\pi_{k}(U), \quad G_{k}=\tau_{k}\left(U_{k}\right), \quad$ and $\quad V=\left\{\left(y_{k}\right)_{k \in K} \in \prod_{k \in K} N_{k}: y_{k} \in\right.$ $\left.G_{k} \cap N_{k}\right\} . V \bigotimes_{k \in K} N_{k}$ will be called a generalized subtensor product of $a$ family $\left(N_{k}\right)_{k \in K}$ with respect to a subdirect product $U$ of $\left(M_{\alpha}\right)_{\alpha \in I}$, and will be denoted by $\otimes_{k \in K} M_{k}$.

RemarK 2. We will demonstrate that $U_{k} \otimes_{\alpha \in J_{k}} M_{\alpha}$ (in (2.2)) can be expressed as $\otimes_{\alpha \in J_{k}} M_{\alpha}$, in generalized definition. For any subset $J$ of $I$, denote $\pi(U)$, where $\pi: \prod_{\alpha \in I} M_{\alpha} \rightarrow \prod_{\alpha \in J} M_{\alpha}, \quad\left(x_{\alpha}\right)_{\alpha \in I} \mapsto\left(x_{\alpha}\right)_{\alpha \in J}$, by $U_{J}$. Consider the special case where $J=K, J_{\alpha}=\{\alpha\}$ for $\alpha \in K, N_{\alpha}=M_{\alpha}$ for $\alpha \in K$, then $V=\left\{\left(y_{\alpha}\right)_{\alpha \in J} \in \prod_{\alpha \in J} M_{\alpha}: y_{\alpha} \in G_{\alpha}\right\}=U_{J}$. Hence

$$
U_{J} \otimes_{\alpha \in J} M_{\alpha}={ }_{V} \otimes_{k \in K} N_{k}={ }_{U} \bigotimes_{k \in K} N_{k} .
$$

$J_{k}$ is a subset of $I$. It follows from (4.4) that ${ }_{U_{k}} \otimes_{\alpha \in J_{k}} M_{\alpha}={ }_{U} \otimes_{\alpha \in J_{k}} M_{\alpha}$.

REMARK 3. The others follow similarly to Remark 2.

5. Application: coproduct of anticommutative algebras. Let $\left(A_{\alpha}\right)_{\alpha \in I}$ be a family of unitary graded algebras over a commutative ring $R$, all admitting the additive group $Z$ of integers as their group of degrees. We shall denote by $A_{\alpha, k}$ the submodule of $A_{\alpha}$ of all homogeneous elements of degree $k$. In case $I$ is finite, $T=\otimes_{\alpha \in I} A_{\alpha}$ is a graded $R$-algebra with $Z$ as its group of degrees [1, pp. 154-156]. But if $I$ is infinite, it is not possible to define a multiplication in $T$ analogously to the finite case so that $T$ is a graded algebra with $Z$ as its group of degrees. However, we shall consider the submodule $A$ of $T=\otimes_{\alpha \in I} A_{\alpha}$ generated by the set of all elements $\otimes a_{\alpha}$ in $T$ such that $a_{\alpha}=1_{\alpha}$ for almost all $\alpha \in I$, where $1_{\alpha}$ is the identity element of $Z_{\alpha}$ for each $\alpha \in I$. 
Definition. For a family $\left(A_{\alpha}\right)_{\alpha \in I}$ of unitary $R$-algebras, a subtensor product with respect to

$$
W=\left\{\left(x_{\alpha}\right)_{\alpha \in I} \in \prod_{\alpha \in I} A_{\alpha}: x_{\alpha}=1_{\alpha} \text { for almost all } \alpha \in I\right\}
$$

in the generalized definition will be called a restricted tensor product and will be denoted by $\otimes_{\alpha \in I}^{w} A_{\alpha}$.

In fact, $A \cong \bigotimes_{\alpha \in I}^{w} A_{\alpha}$, and $A=\sum_{f \in Q} S_{f}$ (direct) by Theorem 5, where $Q=$ $\left\{f \in Z^{I}: f(\alpha)=0\right.$ for almost all $\left.\alpha \in I\right\}$, and $S_{f}, f \in Z^{I}$, is the submodule of $A$ generated by the elements $\otimes a_{\alpha} \in A$ such that $a_{\alpha} \in A_{\alpha, f(\alpha)}$ for all $\alpha \in I$. $S_{f}=0$ for $f \notin Q$, and $S_{f} \cong \bigotimes_{\alpha \in I}^{v} A_{\alpha,(f) \alpha}$.

We now define a multiplication on $A$ so that $A$ becomes a graded algebra. Let $\mu_{\alpha}: A_{\alpha} \otimes A_{\alpha} \rightarrow A_{\alpha}$ be the linearization of the multiplication in $A_{\alpha}$; and for $f, g \in Q$, let $\mu_{\alpha, f, g}$ be the restriction of $\mu_{\alpha}$ to $A_{\alpha, f(\alpha)} \otimes A_{\alpha, g(\alpha)}$; this last module considered as a submodule of $A_{\alpha} \otimes A_{\alpha}$. There is a canonical isomorphism $\omega_{f, g}$ of $S_{f} \otimes S_{g}$ onto $\otimes_{\alpha \in I}^{w}\left(A_{\alpha, f(\alpha)} \otimes A_{\alpha, g(\alpha)}\right)$ which maps the element $\left(\otimes_{\alpha \in I}^{w} a_{\alpha}\right) \otimes\left(\bigotimes_{\alpha \in I}^{w} b_{\alpha}\right)$ to $\bigotimes_{\alpha \in I}^{w}\left(a_{\alpha} \otimes b_{\alpha}\right)$ (cf. (4.2)), considering that $S_{f}$ is canonically identified with $\otimes_{\alpha \in I}^{w} A_{\alpha, f(\alpha)}$, for each $f \in Q$. Let $I$ be totally ordered by $\leqq$, and set $N(f, g)=\sum_{\alpha>\beta} f(\alpha) g(\beta)(N(f, g)$ is properly de-

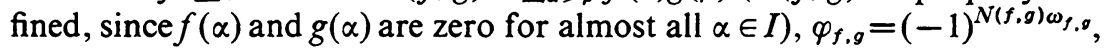
and $\mu_{f, g}=\left(\otimes_{\alpha \in I}^{w} \mu_{\alpha, f, g}\right) \circ \varphi_{f, g}$, where $\otimes_{\alpha \in I}^{w} \mu_{\alpha, f, g}$ is the restriction of $\bigotimes_{\alpha \in I} \mu_{\alpha, f, g}$ to $\bigotimes_{\alpha \in I}^{w}\left(A_{\alpha, f(\alpha)} \otimes A_{\alpha, g(\alpha)}\right)$. Then $\mu_{f, g}$ is a linear mapping of $S_{f} \otimes S_{g}$ into $S_{f+g}$. We may assume that the modules $S_{f} \otimes S_{g}$ are taken to be submodules of $A \otimes A$ and that $A \otimes A$ is their direct sum (cf. (4.3)). Let $\mu$ be the linear mapping of $A \otimes A \rightarrow A$ which extends all the mappings $\mu_{f, g}$. If $a, a^{\prime}$ are any elements of $A$, we set

$$
a a^{\prime}=\mu\left(a \otimes a^{\prime}\right)
$$

this gives a multiplication in $A$, and we have

$$
\left(\bigotimes_{\alpha \in I}^{w} a_{\alpha}\right)\left(\bigotimes_{\alpha \in I}^{w} b_{\alpha}\right)=(-1)^{N(f . g)} \bigotimes_{\alpha \in I}^{w}\left(a_{\alpha} b_{\alpha}\right) .
$$

It can be shown that $A$ is an $R$-algebra, in the same way as [1, pp. 155-156]. For any integer $p$, let $A_{p}=\sum_{s(f)=p} S_{f}$, where $s(f)=\sum_{\alpha \in I} f(\alpha)$. Then $A=$ $\sum_{p} A_{p}$ (direct) is a graded algebra with $Z$ as its group of degrees. Similarly to the proof of [1, Theorem 14, p. 161], it can be shown that if each $A_{\alpha}$ is an anticommutative algebra, then $A=\otimes_{\alpha \in I}^{w} A_{\alpha}$ is also an anticommutative algebra.

THEOREM 6. For a family $\left(A_{\alpha}\right)_{\alpha \in I}$ of anticommutative $R$-algebras, a pair $\left(\otimes_{\alpha \in I}^{w} A_{\alpha},\left(\theta_{\alpha}\right)_{\alpha \in I}\right)$, where $\theta_{\beta}: A_{\beta} \rightarrow \bigotimes_{\alpha \in I}^{w} A_{\alpha}$ is a linear mapping such that $\theta_{\beta}(a)=\otimes a_{\alpha}$ with $a_{\beta}=a, a_{\alpha}=1_{\alpha}$ for all $\alpha \in I, \alpha \neq \beta$, is a coproduct of $\left(A_{\alpha}\right)_{\alpha \in I}$ 
in the category of unitary anticommutative R-algebras and unitary homogeneous homomorphisms of degree zero.

Proof. Similar to the proof of [2, Theorem 1].

\section{REFERENCES}

1. C. Chevalley, Fundamental concepts of algebra, Academic Press, New York, 1956. MR 18, 553.

2. I. Y. Chung, Derivation modules of free joins and m-adic completions of algebras, Proc. Amer. Math. Soc. 34 (1972), 49-56.

Department of Mathematics, University of Cincinnati, Cincinnati, Ohio 45221 